\title{
Near-field Cosmology with the VLT
}

\author{
Steffen Mieske ${ }^{1}$ and Helmut Jerjen ${ }^{2}$ \\ ${ }^{1}$ ESO Garching, Karl-Schwarzschild-Str. 2, 85748 Garching, Germany \\ smieske@eso.org \\ ${ }^{2}$ Mt Stromlo Observatory, Australian National University jer jen@mso.anu.edu.au
}

With the arrival of wide-field imagers on medium-size telescopes (e.g. SkyMapper, Pan-STARRS) and the future co-existence of LSST with the E-ELT, it is worthwhile to evaluate the scientific potential of a CCD camera with $\approx 1$ degree FOV for the VLT. Here we discuss the role that such an instrument could play in resolving persisting fundamental problems in "near-field cosmology".

\section{Science case}

Dwarf galaxies in the local universe are easily studied survivors from the epoch of galaxy formation, and thus preferred targets to establish empirical benchmarks for high redshift cosmological studies, in particular calibrating theories of galaxy formation and interpreting the observed galaxy luminosity function. While $\Lambda \mathrm{CDM}$ predictions for structure formation on large scales agree well with the distribution of baryonic matter (galaxies), it is in the low mass regime where strong discrepancies persist between the expected frequency of low-mass dark matter halos and the number of known dwarf galaxies (e.g. [19], [12], [30], [18]). The most prominent place affected by this so-called substructure crisis is the Local Group, even when accounting for the recently discovered ultra-faint dwarf spheroidals ([24], [31]).

A solution of this problem may well be found in fundamental physics such as warm or self-interacting dark matter ([2], [25]). Alternatively, there is no shortage of astrophysical mechanisms that can diminish the accumulation of baryons in low mass dark matter potential wells: long cooling times for primordial gas in small halos ([5]), galactic winds driven by supernovae and hot stars ([3]), or pressure support against collapse of the intergalactic plasma after reionization $([26],[4])$.

The present picture is further confused because there are a multitude of reasons why the faint end of the galaxy luminosity function, the optical manifestation of the dark matter mass function and completely governed by dwarf galaxies ([1]), could deviate from the simple CDM theory expectation. 
Progress on this fundamental issue is currently limited by observations, not theory as most recent studies of the galaxy luminosity function in Virgo ([20], [29], [23]) and Fornax ([11], [6], [18]) demonstrated that the ambiguity in attributing membership status to cluster/group galaxy candidates is the prime source of uncertainty on the quest to find the accurate shape, slope, and possible turning point of the galaxy luminosity function.

This ambiguity can only be resolved by deriving genuine distances to complete populations of dwarf galaxies.

\section{Galaxy distances from surface brightness fluctuations}

Especially in dense environments, the faint end slope of the galaxy luminosity function is completely determined by the large number of dwarf elliptical galaxies, stellar systems primarily composed of old stars and having an almost featureless morphology (Fig. 1). An intriguing possibility to directly derive distances to such galaxies is the Surface Brightness Fluctuation (SBF) method, whose theoretical framework was developed by [27]. The method quantifies the statistical pixel-to-pixel variation of star counts across a galaxy image with the major technical advantage of working on unresolved stellar populations. Since these variations normalised to the underlying mean galaxy light are inversely proportional to distance (see Fig.2), the SBF amplitude can be used as a distance indicator, once the dependence of the amplitude on stellar content (age, metallicity) is corrected for (e.g. [28]). Jerjen and collaborators ([7], [9], [10], [21] and Mieske et al. ([15], [18]) demonstrated that the method works well for low surface brightness dEs as faint as $\mu_{\mathrm{B}, \mathrm{eff}}=26 \mathrm{mag} \mathrm{arcsec}^{-2}$ and $M_{B}=-10 \mathrm{mag}$, out to distances of $20 \mathrm{Mpc}$ using $8 \mathrm{~m}$ class telescopes.

\section{Statistical analysis of dwarf galaxy properties}

Beyond $30-40 \mathrm{Mpc}$, the SBF method is not efficient anymore for measuring distances to faint dwarf galaxies ([14]). However, near-field cosmology clearly should include the study of low-mass substructures over a range of environments of different scale length: inner-cluster distribution, cluster-to-cluster variations, distribution bias with respect to large-scale filaments and voids. To achieve a proper sampling of these different levels of structure scales, one must extend dwarf galaxy studies out to $\mathrm{z}=0.05$ to 0.1 (see for example [22]), to cover typical filament length scales of a few hundred Mpc. This corresponds to an area on the sky of a few thousand square degrees.

From Fig. 3 it is clear that even with relatively short exposures of a few minutes on an $8 \mathrm{~m}$ class telescopes, low surface brightness galaxies with $M_{V} \sim-11$ mag can be detected. Considering the decrease of angular size with distance, more realistic detection and classification limits for dwarf galaxies are $M_{V} \sim-11$ out to $50 \mathrm{Mpc}$, and $M_{V} \sim-13$ out to $200 \mathrm{Mpc}(\mathrm{z}=0.05)$. 


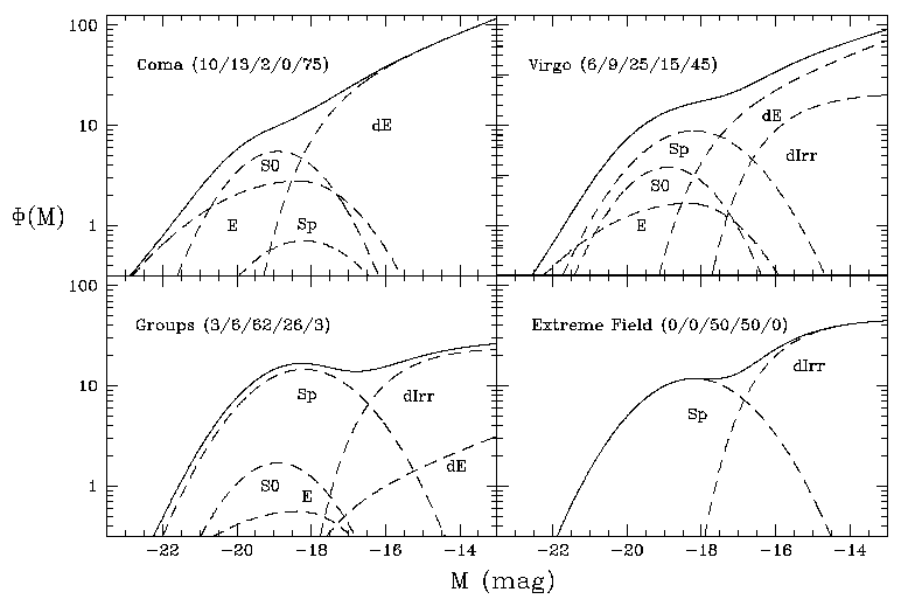

Fig. 1. Galaxy luminosity functions for a range of environments, broken down into different morphological types (Jerjen 2000). The faint end of the luminosity function in dense environments is dominated by early-type dwarf elliptical galaxies.
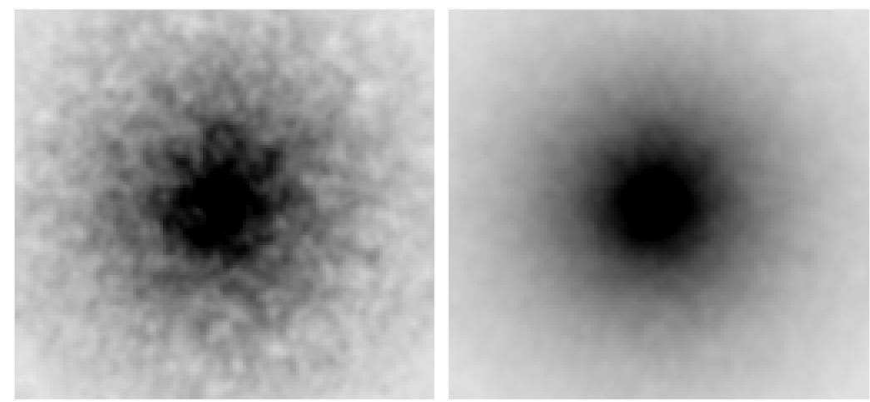

Fig. 2. The principle of SBF distance estimates. Two simulated images of early-type galaxies with implemented surface brightness fluctuations, having identical angular size but different distance ([14]). The galaxy on the right is at a 4 times larger distance than the galaxy on the left giving a smoother apppearance.

Going back to Fig. 1, these magnitude limits are well in the regime where the faint end slope $\alpha$ dominates the shape of the galaxy luminosity function.

Of course, detecting low-surface brightness dwarf galaxy candidates alone is insufficient to derive their absolute luminosity and constructing a fiducial galaxy luminosity function. Therefore, a deeper SBF survey is required covering a few control regions like dense clusters and loose groups to calibrate secondary distance modulus estimators, such as angular size vs. central surface brightness, and colour (e.g. [18]). 


\section{Scientific aims and survey setup}

In summary, we advocate the combination of a smaller scale but deep imaging survey with a shallow imaging survey over a much larger area, in order to study the properties of low-mass galaxies in the nearby universe as a function of environment. The scientific topics that can be addressed by conducting such a near-field cosmology survey are the following:

- Contrast the galaxy luminosity function with the expected 1 CDM mass spectrum. At which luminosity/mass do baryons decouple from dark matter? How does this depend on environment? This will give crucial input for studies on dark matter phase space properties, reionization, feedback, photoionization.

- What are the clustering properties, spatial and angular correlation function of low mass galaxies compared to $\Lambda \mathrm{CDM}$ predictions. What is the origin of satellite galaxies?

- Constrain the anisotropy of dark matter distribution on scales $\leq 100 \mathrm{Mpc}$ (Great Attractor.

- Morphological segregation/transformation, harrassment, as a function of environment.

- Synergy with other scientific areas include the study of globular cluster systems, intra-cluster light, far-field cosmology surveys: ISW, cluster counting, weak lensing, BAO.

While the shallow, dE identification survey could in principle also be done based on data from future surveys with $4 \mathrm{~m}$ class telescopes, the deep distance survey requires the light collecting area of an $8 \mathrm{~m}$ class telescope. This in order to directly derive distances to faint dwarf galaxies with the SBF method. For the deep survey we estimate an area of about 500 square degrees would be sufficient to fully cover the most prominent nearby galaxy clusters $(d \leq 50$ $\mathrm{Mpc}$ ) and a substantial portion of the low density field environment. It would require about one hour of total integration time per pointing shared between $\mathrm{V}$ and $\mathrm{I}$ (or B and $\mathrm{R}$ ) band exposures, in order to derive reliable SBF distances for $\mathrm{d} \leq 50 \mathrm{Mpc}$ and $M_{V} \simeq-14 \mathrm{mag}([14])$. Most of the time will be used for the red filter, which is generally better suited for SBF measurement ([28], [10], [15], [16], [17], [13]). The colour information is used to correct the SBF amplitude for stellar population effects.

With a wide-field imager ( 1 sq degree FOV) at the VLT, one would require about 60 nights of observing time for the deep survey. Assuming a 5000 sq degree coverage for the shallow survey, one would require roughly 100 nights of observing time for 200 seconds exposures in two optical filters V and I (or $\mathrm{B}$ and $\mathrm{R}$ ).

\section{References}

1. Binggeli, B., Sandage, A., \& Tammann, G.A. 1988, ARAA 26, 509 

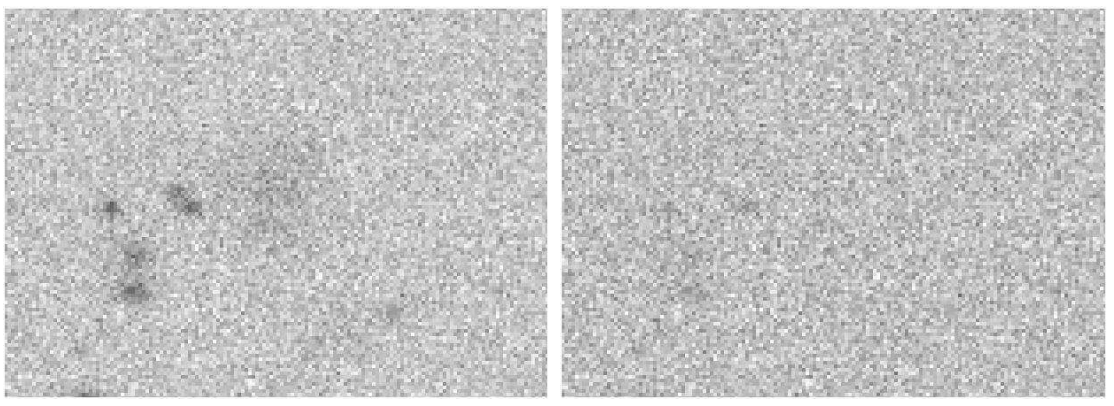

Fig. 3. Here we compare the image obtained from a 200s exposure in V of a dwarf galaxy with $M_{V}=-11 \mathrm{mag}$ at a distance of $20 \mathrm{Mpc}$. The left image corresponds to the VLT mirror size $(8.4 \mathrm{~m})$, the right image to the VST mirror size $(2.6 \mathrm{~m})$. The galaxy is only detectable on the VLT image.

2. Bode, P., Ostriker, J.P., \& Turok, M. 2001, ApJ, 556, 93

3. Dekel, A., \& Silk, J. 1986, ApJ 303, 39

4. Gnedin, N.Y. 2000, ApJ 542, 535

5. Haiman, Z., Thoul, A.A., \& Loeb, A. 1996, ApJ 464, 523

6. Hilker, M., Mieske, S., \& Infante, L. 2003, A\&AL, 397, L9

7. Jerjen, H., Freeman, K.C., Binggeli, B. 1998, AJ 116, 2873

8. Jerjen, H., Encyclopedia of Astronomy and Astrophysics, ed. Paul Murdin, Bristol: Institute of Physics Publishing, 2000

9. Jerjen, H., Freeman, K.C., Binggeli, B. 2000, AJ 119, 166

10. Jerjen, H. et al. 2001, A\&A 380,90

11. Kambas, A., Davies, J. I., Smith, R. M., Bianchi, \& S., Haynes, J. A., 2000, AJ 120,1316

12. Klypin, A., Kravtsov, A. V., Valenzuela, O., \& Prada, F. 1999, ApJ, 522, 82

13. Mei, S. et al. 2005, ApJ, 625, 121

14. Mieske, S., Hilker, M. \& Infante, L. 2003a, A\&A, 403, 43

15. Mieske, S. \& Hilker, M. 2003b, A\&A 410, 445

16. Mieske, S., \& Hilker, M. \& Infante, L. 2005, A\&A, 438, 103

17. Mieske, S., Hilker, M. \& Infante, L. 2006, A\&A, 458, 1013

18. Mieske, S., Hilker, M., Infante, L., Mendes de Oliveira, C. 2007, A\&A, 463, 503

19. Moore, B., Ghigna, S., Governato, F. et al. 1999, ApJL, 524, 19

20. Phillipps, S. et al. 1998, ApJ, 493, L59

21. Rekola, R., Jerjen, H. \& Flynn, C. 2005, A\&A 437, 823

22. Rosenbaum, S.D., \& Bomans, D. 2004, A\&A, 422L, 5

23. Sabatini, S. et al. 2005, MNRAS, 357, 819

24. Simon, J. D., \& Geha, M. 2007, ApJ, 670, 313

25. Spergel, D.N. \& Steinhardt, P.J. 2000, Physical Review Letters 84, 3760

26. Thoul, A.A. \& Weinberg, D.H. 1996, ApJ 465, 608

27. Tonry, J.L. \& Schneider, D.P. 1988, AJ 96, 807

28. Tonry, J.L. et al. 2001, ApJ 546, 681

29. Trentham, N. \& Hodgkins, S. 2002, MNRAS 333, 423

30. Trentham, N., \& Tully, R.B., 2002, MNRAS 335, 712

31. Walsh, S.M., Jerjen, H., Willman, B., 2007, ApJL, 659, 121 